\title{
Large-Separation Lensed Quasars in the SDSS
}

\author{
Masamune Oguri ${ }^{1}$ \\ ${ }^{1}$ Department of Physics, University of Tokyo, Tokyo, 113-0033, JAPAN email: \\ oguri@utap.phys.s.u-tokyo.ac.jp
}

\begin{abstract}
The existence of lensed quasars with splitting angles larger than $7^{\prime \prime}$ has been predicted on the basis of the cold dark matter model. We searched for these large-separation lensed quasars from the spectroscopically classified $\sim 40,000$ quasars obtained by the Sloan Digital Sky Survey (SDSS), and succeeded in discovering the first lensed quasar by a cluster of galaxies, SDSS J1004+4112: It consists of four images, and the maximum separation is $14.62^{\prime \prime}$. Here we describe the discovery and the follow-up observations of this system, as well as the latest results of the large-separation lens search in the SDSS. We also present the new statistical model which fully incorporates non-sphericity of dark halos, and compare the theoretical predictions with the SDSS results.
\end{abstract}

\section{Introduction}

Image separations between multiple images play a central role in strong gravitational lensing of quasars, because image separations are mainly determined the mass of lens objects; thus the image separation distribution of lensed quasars directly reflects the hierarchical structure of the universe. Small separation lenses, with image separations $\theta \sim 1^{\prime \prime}$ that mean the lens object would be massive galaxies, are quite familiar since the first discovry in 1979; currently $\sim 80$ small-separation lensed quasars are known (Figure 1 ), and they offer unique probes of structure of distant galaxies as well as cosmological parameters. On the other hand, another population of large-separation lenses (say, $>10^{\prime \prime}$ ) is also theoretically expected in the framework of the Cold Dake Matter (CDM) model. However, the large-separation lensed quasars have been much less familiar because of the smaller lensing probability; numerical simulations based on the CDM have shown that dark halos (that are good approximation of clusters of galaxies) are less centrally concentrated than a singular isothermal distruibution that can be used to describe the mass distribution of galaxies.

Althogh the expected lensing probability of large-separation lensing is small, progress in the observational side begins to make it possible to find such lenses. For instance, radio survey of the Cosmic Lens All Sky Survey (CLASS; Phillips et al. 2001) has contain 10, 000 radio sources, and the lack of large-separation lenses turned out to be marginally consistent with the CDM prediction (Keeton \& Madau 2001). Therefore it has been expected that larger surveys may be able to find the first example.

\section{Discovery of SDSS J1004+4112}

To find large-separation lenses, we use both the imaging and spectroscopic data of the Sloan Digital Sky Survey†. Currently it contains spectroscopically classified $\sim 40,000$ quasars at $z>0.6$, about four times larger than the CLASS sample. For each quasar, we

$\dagger$ http://www.sdss.org/ 


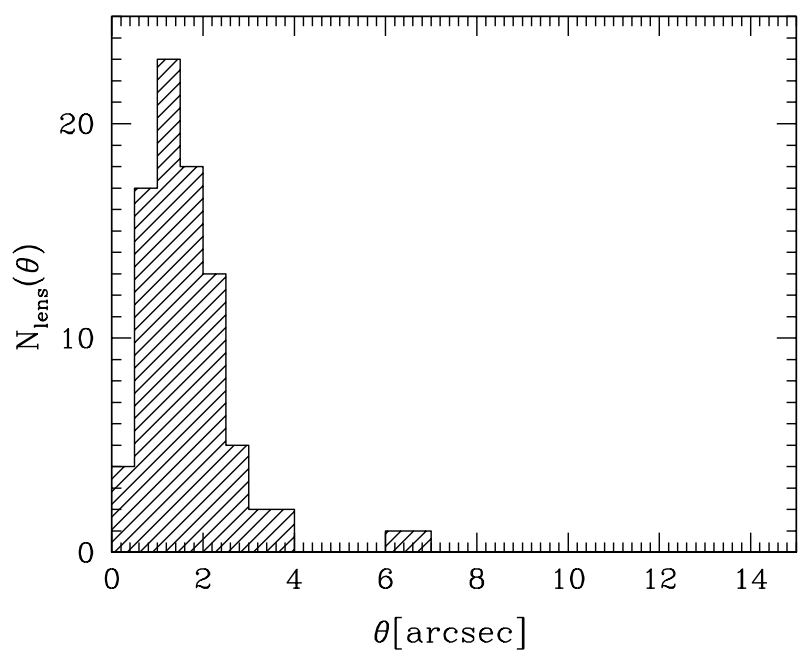

Figure 1. The image separation distribution of all gravitationally lensed quasars discovered so far. Lenses discovered in the SDSS are not included.

look for nearby stellar objects that have similar color to the quasar. Since the fiber for spectroscopy has an unnegligible size that corresponds to $55^{\prime \prime}$, basically the objects do not have the SDSS spectra; thus we need spectroscopic follow-up observeations to check their redshifts. We note, however, that the five borad-band imaging of the SDSS is quite essential to select lens candidates quite efficiently.

SDSS J1004+4112 (the maximum image separation of $\sim 15^{\prime \prime}$ ) was identified as a good lens candidate using the method described above. The fold-type quadruple configuration makes it already clear that it is a true gravitationa lens system, and indeed the spectroscopy at ARC showed that all four components have the same redshift, $z=1.734$. Nevertheless we carefully examined the lensing hypothesis by conducting further spectroscopic observation at Keck telescope and the imaging/spectroscopic observation at Subaru telescope.

It turned out that the data strongly support for the lensing hypothesis (see Figure 2): Spectra of four stellar components taken at Keck show reasonable agreement, and the deep image taken with Subaru revealed a cluster of galaxies that supposed to be responsible for the large image separation. We found that the lensing cluster is at $z=0.68$ from spectroscopies of central three galaxies. For more detailed analyses of this system, please refer to Inada et al. (2003) and Oguri et al. (2004).

\section{Follow-up Observations of SDSS J1004+4112}

The uniqueness of the lens system urges us to conduct further follow-up observations. First, to resolve the large difference of emission lines between component $\mathrm{A}$ and the other components we took seven epoch spectra of components A and B. Surprisingly, we have detected the enhancement of the blueward of the emission line and its fading; by combining the timescale and the predicted time delays between $\mathrm{A}$ and $\mathrm{B}$, we concluded that the enhancement was caused by microlensing of the broad emission line region, rather than the intrinsic fluctuation of the spectrum of the source quasar (Richards et al. 2004).

We also took the Hubble Space Telescope (HST) images of SDSS J1004+4112, and obtained two interesting results. First, we have found the lensed host galaxy of the source 


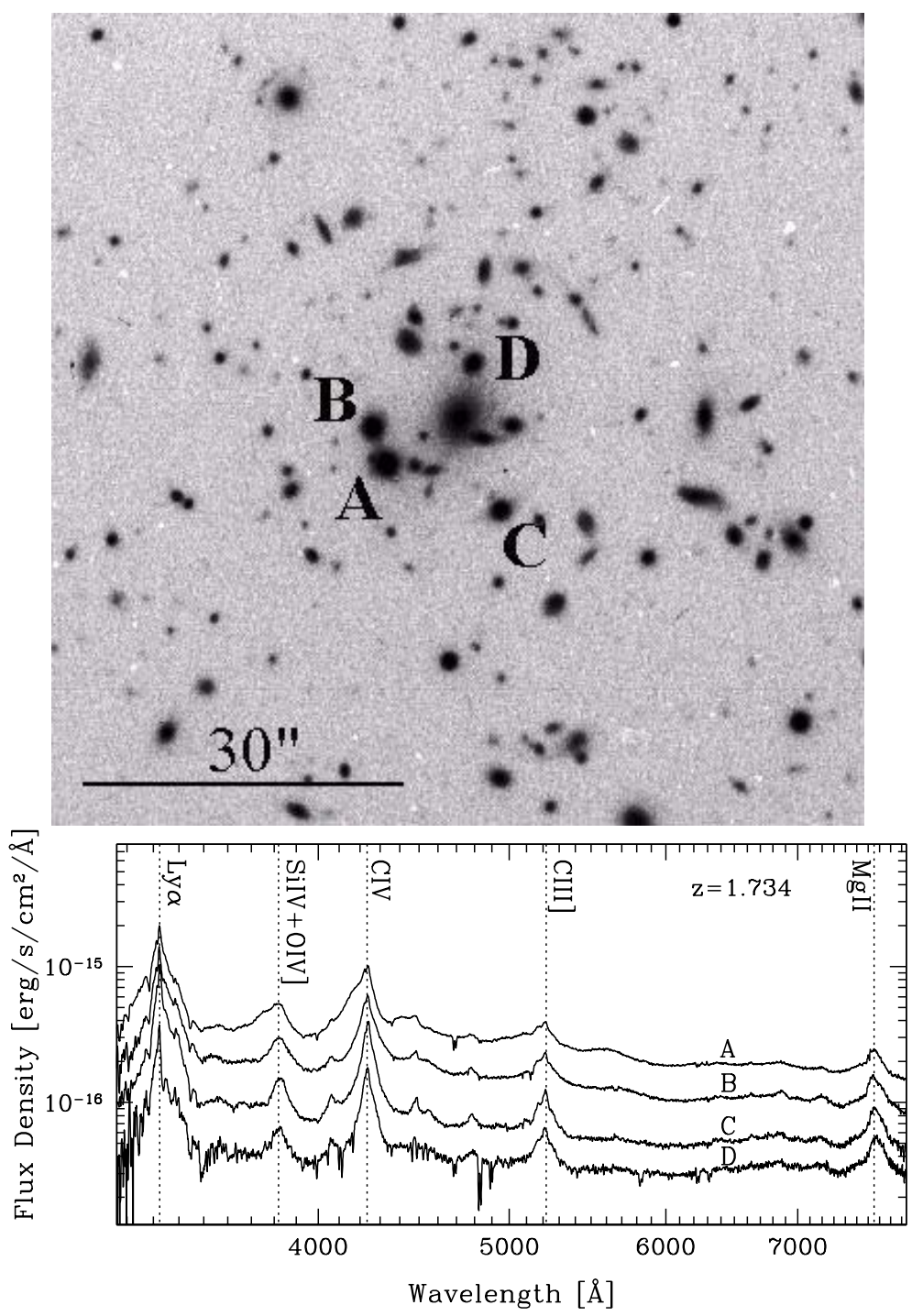

Figure 2. Upper: Deep $i$-band image around SDSS J1004+4112 taken at Subaru telescope. Lower: Spectra of four stellar components of SDSS J1004+4112 taken at Keck telescope. See Oguri et al. (2004) for more details.

quasar. The lensed host galaxy is seen in the ACS image, but is more prominent in the NICMOS image (see Figure 3). Second, we discovered a stellar object near the center of the galaxy G1 (see Figure 4), which may be the fifth image; such central odd image can offer unique probe of the core of the lens object (e.g., Winn, Rusin \& Kochanek 2004). We need further follow-up observations to test this fifth-image hypothesis.

Another imoprtant follow-up observation is monitoring of the system for measuring time delays between images. Currently time delays are very useful to put additional constraints on the lens potential, rather than to determine the Hubble constant (Oguri 

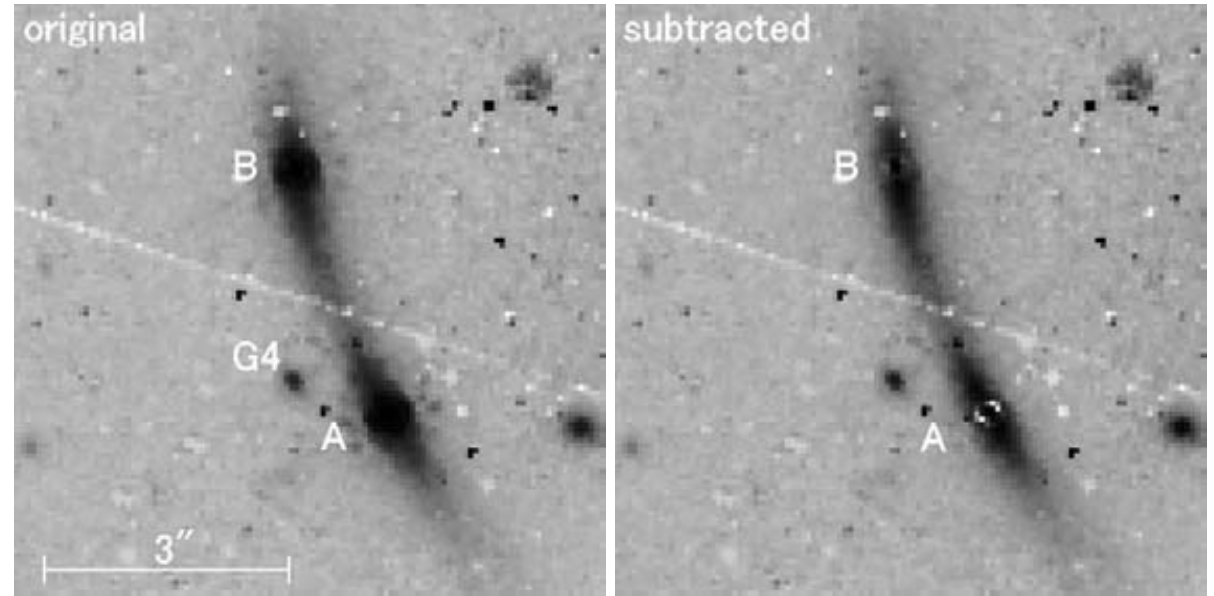

Figure 3. Left: The NICMOS image of SDSS J1004+4112. The central line at an angle is the bad column. Right: The NICMOS image after subtracting the quasar components. The lensed host galaxy is seen more prominently. See Inada et al. (2004) for more details.

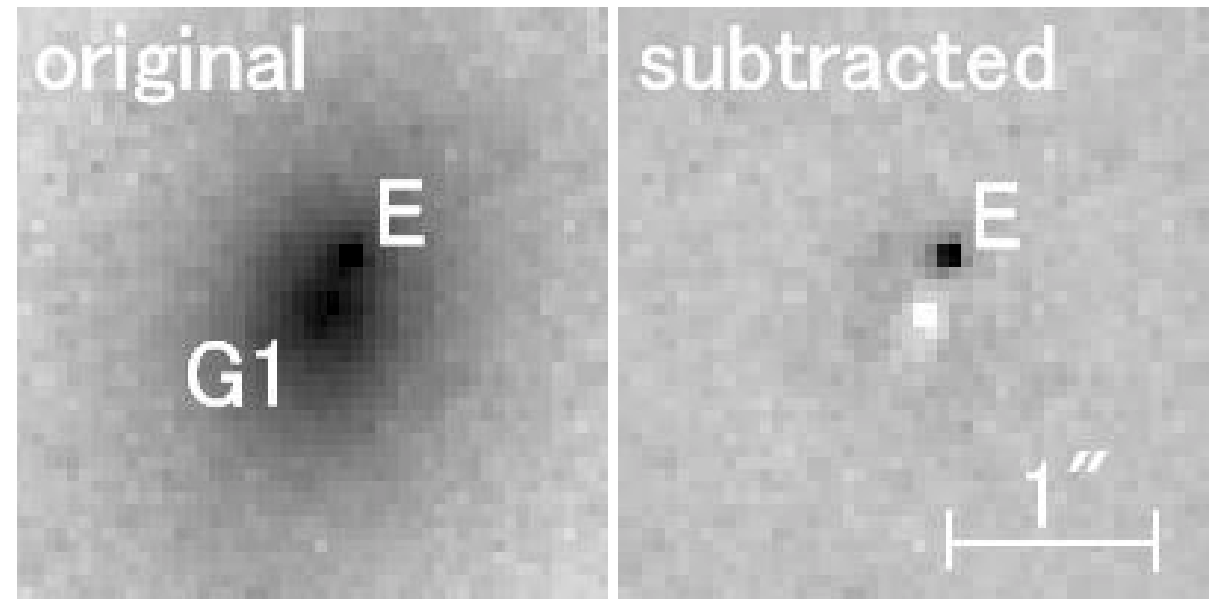

Figure 4. Left: The ACS image of the central galaxy G1. Right: The ACS image after subtracting the galaxy G1. The residual image clearly shows a stellar object near the center of G1, which may be the fifth image. See Inada et al. (2004) for more details.

et al. 2004). To measure time delays, we have started to monitor it with MAGNUM telescope $\dagger$.

It is very attractive to conduct observations at other wavelength; we observed the system with Nobeyama 45-meter radio telescope to find the Sunyaev-Sel'dovich signal of the lensing cluster, and now reducing the data. We plan to take the image with Chandra to study both the lensed quasar and the lensing cluster.

\section{SDSS Large-Separation Lens Search: Current Status}

The SDSS is still ongoing, thus the number of quasars used to search for large separation lensed quasars is also increasing. We have discovered many good lens candidates in

$\dagger$ The 2-meter optical/IR telescope dedicated for minitoring AGN/QSO. The telescope is owned by the University of Tokyo. 

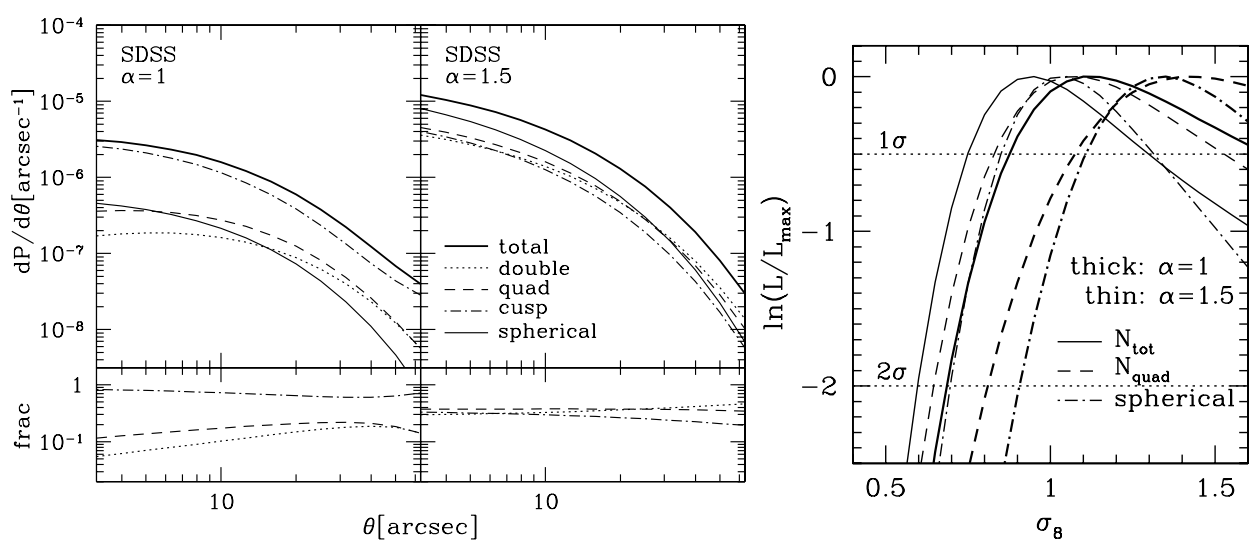

Figure 5. Left: Lensing probabilities and image multiplicities for SDSS quasars at redshifts $0.6<z_{\mathrm{S}}<2.3$. The left panels are for $\alpha=1$ and the right panels for $\alpha=1.5$, where $\alpha$ is inner slope of the density profile $\rho \propto r^{-\alpha}$. Right: Maximum likelihood estimates for $\sigma_{8}$ from combining the discovery of SDSS J1004+4112 in SDSS with the lack of large-separation lenses in CLASS. In making predictions for SDSS, we consider two cases: the appropriate prediction could be the total number of lenses (solid lines); or since SDSS J1004+4112 is a quad the appropriate quantity could be the number of quadruples (dashed lines). The likelihoods for $\alpha=1$ and 1.5 are shown by thick and thin lines, respectively. Results for spherical halos are also shown by dash-dotted lines for reference.

the SDSS, including those that have possible lens galaxies. However, still we can't find the second example of large-separation lenses; some of candidates turned out to have the same redshifts, but the shapes of spectra were very different between components. For those quasar pairs, we conducted imaging observations to look for lens objects (which are supposed to be clusters of galaxies), but it was not so successful. As a result, we have found tremendous number of physical quasar pairs, which might be a clue to the formation of quasars (Hennawi et al. 2004).

\section{Statistics of Large-Separation Lensed Quasar with Triaxial Halos}

Is the discovery of the quadruple lens SDSS J1004+4112 in the current SDSS quasar sample consistent with the theoretical expectation? To answer this, we computed lensing probabilities with lens objects modeled by triaxial halos, motivated by the recent study (Oguri, Lee \& Suto 2003) that showed the triaxiality does affect arc statistics significantly. The big advantage of such non-spherical modeling is that it allows us to predict image multiplicities; since image multiplicity depends both on the degree of non-sphericity and the central concentration, it will offer us new insight on the shape of clusters of galaxies.

The image separation distributions of lensing probability and image multiplicity are shown in Figure 5. It is clear from this Figure that (1) the triaxiality enhances the probability for large separation lensed quasars by several factors (2) image mulitiplicity strongly depends on the inner slope of the density profile, $\alpha(3)$ the model predicts the significant fraction of lensing by naked cusp that is very rare in small separation lensing. These predctions should be tested against observations.

We compare the model predction with the discovery of SDSS J1004+4112. First, we compute the fraction of quadruple lensing in the SDSS quasar sample, and find that the fraction is quite significant $(>20 \%$ ). Thus the fact that we discovered a quadruple lens first is fully consistent with the theoretical prediction. In contrast, many lens candidates discussed so far (e.g., Miller et al. 2004) are double that is not dominated in theory. This 
means that most of them are not likely lenses, if the theory is correct. Next we constrain $\sigma_{8}$ from the discovery of one in the SDSS and the lack of large separation lens in the CLASS, as also shown in Figure 5. Thus our conclusion is that the CDM model with $\sigma_{8} \sim 1$ well explain the observed lensing probability. See Oguri \& Keeton (2004) for more details.

\section{Summary}

We have presented the large separation lensed quasar search project in the SDSS; among the current $\sim 40,000$ quasars, we have discovered the first large separation lensed quasar SDSS J1004+4122. Since it shows several interesting features, many follow-up observations of this system have been conducted/planed. We have also computed lensing probabilities and image multiplicities using triaxial halo model, and found that the discovery of the SDSS J1004+4112 is consistent with the CDM predictions.

\section{Acknowledgements}

This work is based on the collaborations with the SDSS strongly lensed quasar search team, and I'd like to thank all the members for the exciting collaborations. Particularly I am grateful to Naohisa Inada, Bart Pindor, Joe Hennawi, Chuck Keeton, Michael Gregg, Bob Becker and Gordon Richards for their significant contributions to the SDSS large-separation lens search project. MO is supported by JSPS through JSPS Research Fellowship for Young Scientists.

\section{References}

Hennawi, J. F., et al. 2004, in preparation.

Inada, N., et al. 2003, Nature 426, 810.

Inada, N., et al. 2004, in preparation.

Keeton, C. R., \& Madau, P. 2001, ApJ (Letter) 549, L25.

Miller, L., Lopes, A. M., Smith, R. J., Croom, S. M., Boyle, B. J., Shanks, T. \& Outram, P. 2004, MNRAS 348, 395.

Navarro, J. F., Frenk, C. S. \& White, S. D. M. 1997, ApJ 490, 493.

Oguri, M., Lee, J. \& Suto, Y. 2003, ApJ 599, 7.

Oguri, M., et al. 2004, ApJ 605, 78.

Oguri, M. \& Keeton, C. R. 2004, ApJ 610, 663.

Phillips, P. M., et al. 2001, MNRAS 328, 1001.

Richards, G. T., et al. 2004, ApJ 610, 679.

Winn, Rusin \& Kochanek 2004, Nature 427, 613. 